\title{
Chemical Safety Management Training Hub for Downstream Users \& Distributers of Chemicals
}

\author{
THEODOR HARATAU, IOANA PRECUP, SIMONA STEFANESCU* \\ Romtens Foundation, 107, Bl. 24C, Sc. A, Et. 3, Ap. 11, Ştirbei Voda Str., 010108, Bucharest, Romania
}

\begin{abstract}
Chemical Safety Management Training Hub for Chemicals Users/ChemSM-HUB - project funded under Erasmus + Programme - has been tailored to meet the training needs related to legal provisions under REACH \& CLP Regulations of employees responsible for CSM among downstream users $(D U)$ and distributers $(D)$ of chemicals. The strategic objectives proposed by the project were to increase awareness of relevant professional communities on the new European legislation on CSM, strengthen the competencies of the target groups in the field of CSM, and enhance the ability of trainers to create and deliver vocational training. The design and concept of the training hub and the training materials have been established based on a training needs assessment, which also provided the necessary information to define the training objectives, the format, and the type of materials to be developed. To ensure high quality of the training, but also the availability in a user-friendly format, the training materials and the training hub have been developed and improved progressively as part of a consortium peer-review process during the joint-staff training organized in Romania according to the format of Erasmus + Programme - KA202 Strategic Partnerships for vocational education and training. Also, the materials created have been subjected to pilot testing during the blended mobility training of VET learners within Erasmus + Programme - which, according to the new circumstances imposed by Sars-Cov-2 pandemic has been organized strictly online using an integrated approach of a webinar application and the ChemSM-HUB e-learning platform. According to the feedback received in both cases - during peer review and the pilot training - materials and the hub have been subject to various changes and resulted in a comprehensive training package and, respectively, a training hub that facilitates individual study and certification of the DU \& D of chemicals, and provides access to up-todate information in a concise and structured format.
\end{abstract}

Keywords: REACH, CLP, regulation, chemical safey management, vocational training

\section{Introduction}

The chemical industry remains one of the largest production sectors in Europe, with $€ 3,347$ billion sales in 2018, although there has been recorded a decline in the overall number of companies in most chemical industry subsectors since 2009. At the global level, Europe is the second-largest chemicals producer in the world [1], and is characterized by geographical concentration as approximately $70 \%$ of chemical companies are located in only six EU countries - France, Germany, Italy, Spain, Poland, the UK - [2], and by the predominance of small and medium-sized enterprises (SMEs) within all EU chemical industry subsectors where the percent of SMEs revolves around 96 to $98 \%$ out of the total number of companies [2].

The context in which these enterprises operate has been transformed with the Regulation (EC) No 1907/2006 of the European Parliament and of the Council on the Registration, Evaluation, Authorisation and Restriction of Chemicals (REACH), approved in 2006. The REACH Regulation produced numerous changes related to chemical safety management in EU companies, amended or repealed all previous regulations and introduced an integrated system of registration, evaluation, authorisation, and restriction of chemicals produced or supplied in the EU [3]. Beyond the advancement towards better management of risks and increasing substitution of substances of high concern in the late years, the fundamental novelty of the legislation represents the transfer of the burden of responsibility for chemical safety from

*email:office@romtens.ro 
the Member States to the companies. Hence, after the entry into force of the REACH Regulation, SMEs have the same responsibilities as large companies and cannot be exempted from any chemical safety obligations [4], although most of these cannot afford to hire a specialist in the field of chemical safety management (CSM).

In this context, the European Chemicals Agency (ECHA) places a special focus on helping the SMEs, by providing guidance and information tools, to plan and submit applications of authorizations under the standardized format [3]. Moreover, ECHA mitigates also for the safe use of chemicals by supporting and initiating other pieces of legislation, among which the Regulation (EC) No 1272/2008 of the European Parliament and of the Council on the Classification, Labelling and Packaging of substances and mixtures (CLP) which came into force in January 2009 and amended by the Regulation (EU) 2019/521. By advancing the importance of having harmonized requirements and criteria for classification, labelling and packaging ECHA underlines its value to be committed to well-being through establishing the context for companies to ensure and prove the safe use of substances and mixtures so that to minimize as much as possible the adverse effects on human health and the environment [5]. Given the legal circumstances, the challenges for companies prevail not only in providing data to ECHA, but also in collecting hazard related quality data about the substances, the extent to which workers or consumers are exposed to these hazards, as well as the risks related to the uses of substances [6]. Moreover, depending on their place in the supply chain, actors will have different obligations. Manufacturers and importers have to provide information to their customers on how to use the substances, while customers (i.e. downstream users) must give information back to the manufacturer on how they use the chemicals - information to be then included in the registration dossier [7]. The new principle of 'no data, no market' (Article 5) introduced by REACH is that only substances registered with ECHA are lawful [2].

These new insights of legislation triggered two interdependent actions - one related to the companies need to improve the quality of the information in their safety data sheets and the second one related to the need to acquire more in-depth information \& training when it comes to legislative provisions and the best way to implement them. The latter became even more stringent for the SMEs with scarce resources for specialists and tailored training needed to cope with the new requirements.

Starting from these preconditions, the project - Chemical Safety Management Training Hub for Chemicals Users/ChemSM-HUB, funded under Erasmus+ Programme, has been tailored to meet the training needs related to legal provisions under REACH \& CLP Regulations of employees responsible for CSM among downstream users (DU) and distributers (D) of chemicals - including professional and industrial chemical users, formulators, fillers, article producers, craftsmen, service providers, distributors of chemicals, as well as occupational safety and health (OSH) specialists and state authorities, like sanitary inspection and labor inspection. [4]. The training aims to help the target groups better understand and observe their legal duties. The strategic objectives proposed by the project are to increase awareness of relevant professional communities on the new European legislation on CSM, strengthen the competencies of the target groups in the field of CSM, and enhance the ability of trainers to create and deliver vocational training. The project consortium is represented by 5 institutions from 4 EU countries, namely: Nofer Institute of Occupational Medicine (NIOM) Poland, Lodz University of Technology (TUL), Poland, Oekopol - Institute for Environmental Strategies (Oekopol GmbH), Germany, The Institute of Preventive Medicine, Environmental and Occupational Health (Prolepsis) Greece, and Romtens Foundation, Romania.

\section{Approaches for developing the training hub}

By establishing first the availability of CSM training in partner countries, the project followed the stages of gradual development of a training hub as a training platform aiming to provide free access to materials, tools, guidance, and sources of information to the target groups defined by the project. This would ensure that irrespective of the workplace or residence, the beneficiaries could have available means of information and training, as well as guidance for the consultation of other official sources and 
the design of own training adapted to specific needs of the companies. Moreover, to ensure quick access to all facilities of the training hub, a mobile application for android devices has also been developed.

The design and concept of the training hub and the training materials have been established based on a training needs assessment, which also provided the necessary information to define the training objectives, the format, and the type of materials to be developed. Moreover, the design and concept have been enriched by integrating into the analysis aspects related to the target group definitions and area of responsibility, the profile of future trainees (educational profile, occupation, expectations, skills, etc.), and by desktop review to identify useful sources of data which can be used by chemical safety experts.

Following the needs assessment, the curriculum of each of the training modules and the logic sequence of activities has been established, setting at the same time the duration and possible training design for each level. This process resulted in developing a training package that incorporates:

- An introductory module, elaborated with the purpose to serve as an introduction in the field of CSM for the future trainees by covering the very basic information related to the legislation in the field (REACH \& CLP). This module has been intended for individual study.

- A basic module, containing four sessions covering different topics, each of these reduced to a basic knowledge level covering the essentials for the newcomers in the field (topics related to REACH, CLP, Safety Data Sheets, and ECHA).

- An advanced module, structured in seven different sessions dedicated to trainees who are more experienced in the field and covering details on the safety data sheets, restrictions and authorizations processes, classification of mixtures - physical hazards, health hazards, and environmental hazards, and not the last - labeling.

As part of the training package, the partners have also foreseen and developed two very comprehensive and consistent materials, in particular, the "Training Pattern for Trainers" and the "Pocket Guide for Downstream Users and Distributors of Chemicals - REACH \& CLP Essentials". Both training materials have been made available both in printed and electronic format. The "Training Pattern for Trainers" is designed as a road map for the chemical safety experts who want to organize training sessions covering chemical safety management based on the provisions of REACH \& CLP Regulations, while the pocket guide represents a source for downstream users and distributors of chemicals to help them meet the obligations imposed on companies under the REACH and CLP Regulations and become acquainted with the basic concepts of CSM as well as basic REACH and CLP procedures. The need to develop the "Pocket Guide for Downstream Users and Distributors of Chemicals - REACH \& CLP Essentials" has arisen from the results of the survey conducted by the project team, which revealed that many companies, especially small and micro companies, still have many problems in understanding the REACH and CLP Regulations or interpret them incorrectly. There is a widespread belief that these regulations are too complex and, that the legal rules included are subject to continuous change over time which causes additional problems, hence companies have problems defining their legal obligations under the REACH and CLP Regulations.

All the above-mentioned materials have been made available free of charge in the national languages of the 4 partner countries represented in the project - Poland, Germany, Greece, and Romania.

\section{Approaches for evaluating the training materials}

To ensure high quality of the training, but also the availability in a user-friendly format, the training materials and the training hub have been developed and improved progressively and in parallel with comments and changes suggested as part of a consortium peer-review process during the joint-staff training organized in Romania according to the format of Erasmus+ Programme - KA202 Strategic Partnerships for vocational education and training.

Also, the materials created have been subjected to pilot testing during the blended mobility training of VET learners within Erasmus+ Programme - which, according to the new circumstances imposed by Sars-Cov-2 pandemic has been organized strictly online using an integrated approach of a webinar application and the ChemSM-HUB e-learning platform. The unfolding of the training has been conducted 
very similar to a face-to-face course as it took a significant time commitment from the trainees' part of around eight (8) hours per day in the online classroom working on the course materials together with the trainers.

The structure of the CSM Training corresponded to 8 training sessions consisting of theoretical and practical units. Each theoretical unit has been followed by a practical unit conducted under the supervision of the trainer. The pilot training program has covered the following topics (1) - Introduction to the REACH and CLP Regulations, (2) - Restrictions under REACH Regulation, (3) - Authorisation under REACH Regulation, (4) • Safety Data Sheet for a hazardous chemical substance/mixture under REACH, (5) - Classification of mixtures in terms of physical, health and environmental hazards under CLP Regulation, (6) • Labeling of chemical substances and mixtures under CLP Regulation.

The assessment of the pilot training course aims to determine the attainment of specific Objective 2 "Enhancing the competences in the area of chemical safety management of downstream users (DU) and distributors (D) of chemicals across four countries". The concept proposed derives from the four-level evaluation model of training courses proposed by Kirkpatrick which structures the evaluation of training around four sequenced tiers such as Reaction, Learning, Behavior, and Results [8]. Reaction can be translated into what we call satisfaction assessment and it is a one-time evaluation, very common, and usually integrated into the training course evaluation. Learning assessment is defined under three other sub-dimensions namely the extent to which attitudes have been changed, the extent to which knowledge has been improved, and the extent to which skills have been acquired. Behavior assessment revolves around the extent to which behavior change has occurred as a result of attending the training program. However, other determinants impact behavioral change and might be responsible for changes in performance [9], hence in generating results. Which brings the Results assessment into focus which can be defined as the final results that occurred because the participant attended the program. This is indeed the most difficult and resource-intensive type of evaluation as it usually entails the support of on the ground teams of evaluators. Nevertheless, according to Phillips ROI Model, which extends and improves the Kirkpatrick's model, there is also and additional fifth $\left(5^{\text {th }}\right)$ step, namely the return on investments (ROI) which integrates cost benefit analysis to help determine if the financial resources invested generate any returns [9].

By considering all the constraints applicable to the ChemSM-HUB training course and the project implementation timeline which does not permit to measure neither behavioral change in time, nor results, the approach proposed has been to perform two of the four types of evaluation from Kirpatrick's model:

1.Reaction is the evaluation of satisfaction of the downstream users (DU) and distributors (D) of chemicals to identify how the unfolding and structure of the course have been perceived and how the participants relate to the training content. For this type of evaluation, a post design has been considered, hence the developed questionnaire has been filled in by the training participants only one time, at the end of the training. The information gathered has been collected for the process of improving and adjusting the training materials to develop the final version of the training modules.

2.For Learning only one sub-dimension has been considered, namely to determine the contribution of the training course in increasing knowledge. For this type of evaluation, a pre/post design has been considered by developing an Initial Knowledge Check questionnaire to assess the participants' level of knowledge on the elements of CSM before attending the training. For the post-evaluation a Final Knowledge Check questionnaire has been prepared, comprising the same questions as the Initial Knowledge Check questionnaire - to determine and measure the effectiveness of the training. The sum of points received for each question has represented the evaluation of a participant pre/post-training and the maximum number of points has been set to 100 , each of the 25 questions having 4 points assigned.

Besides the evaluation of the training from the participants' perspective, it has been considered important to address the evaluation of the training from the viewpoint of the trainers. Therefore, a checklist has been developed for the trainers to be filled in at the end of the training course. The process of filling in the checklist has taken place after the training course so as to be able to capture all the relevant information. 
The data collection process has been carried out in September 2020 and October 2020, given the unfolding of the pilot training, from 28 September 2020 to 02 October 2020.

For the satisfaction assessment, a number of 15 feedbacks have been received. The analysis focuses mainly on descriptive statistics, considering the objectives of the evaluation and the nature of the questionnaire.

For the Initial Knowledge Check questionnaire 26 responses have been received, given that 26 invitees have accepted the online training invitation. In the end, not all have been able to join the training, and only 15 trainees have provided feedback during both pre- and post- evaluation. Hence for the assessment of the knowledge attainment (pre/post evaluation), the dataset comprises 15 questionnaires; as the others have been excluded from the analysis because of missing data for the Final Knowledge Check. No missing values have been recorded for the questions mainly because all questions have been set as mandatory - a thing which can be easily controlled when applying online questionnaires/tests. The analysis of the responses has been focused both on comparing overall pre- and post-training scores, and comparing the number of respondents answering each one of the questions correct pre- and posttraining. From a statistical point of view, an improvement of knowledge has been considered as significant, there has been a statistically significant difference between pre- and post- answers based on Wilcoxon non-parametric tests. The Wilcoxon test has been applied to test whether there is a statistically significant difference in respondents' overall scores pre- and post-evaluation.

The data collected through the checklist for trainers has been compiled and analyzed in conjunction with the data collected from the trainees. In total three (3) checklists have been filled in by the three (3) trainers.

Hereinafter the terms respondents, trainees, participants refer to those 15 respondents who provided feedback to the satisfaction questionnaire and responded to both pre \& post training evaluation.

\section{Results and discussions}

A number of 15 trainees joined the training representing companies which are downstream users (DU) and distributors (D) of chemicals. Most of the respondents work at multi-national companies (6) and in national or international public institutions (5) and only 4 respondents represent national companies. The training has been of interest to both young professionals with less experience in practice, and to more experienced professionals, as the number of years in practice varies from less than a (1) year to 20 years, but on average, a trainee has about 7 years in practice - only 4 of the respondents have more than 7 years' experience. The distribution of respondents by age reflects that most of them are 30 to 39 years and 40 to 49 years old (11 out of 15 respondents). The demographic information should be considered only relative to the pilot training and cannot be held as a determinant for choosing to participate in a training chemical safety management, nor can it be considered representative for the experts in the field of chemical safety management.

From the trainees' satisfaction assessment, it appears that for almost all participants (14 out of 15) the overall online training experience has been a positive one and would recommend the ChemSM-HUB training course to other colleagues should it be made available outside the project. The overall objective of the training - to help you understand the legal duties and the EUR legislation on CSM - has been considered to be achieved, showing that the objective set has been realistic. Considering the practical application of the training content, also 14 out of 15 respondents intend to apply in practice the knowledge gained during the training sessions. This highlights that trainees related to the content of the training as rooted in the work context of CMS and that the trainees could link the information to their own work experience.

Considering the content-related aspects, all have been positively assessed by most of the participants, but it stands out that all trainees agree that the content is relevant in addressing CSM related issues, and most of them (about 13 out of 15) agree that it is adequate for the scope of CSM, is useful for applying in practice and is clear in presenting the information. Nevertheless, some respondents consider the content to be less comprehensive as to what CSM implies and to have a smaller coverage of various 
perspectives of when and how to apply CSM tools. This was more or less an expected answer from the participants, as the CSM field is a very broad one and no matter how comprehensive the authors would have liked to elaborate the materials, there are still much to be addressed - many sources being though included on the ChemSM-Hub.

As for the training hub, the feedback received shows that it provides a good user experience enabling easy navigation and a clear and logical structure of the materials which makes the training materials easy to find and the exercises easy to process (14 out of 15 respondents). Although 13 out of 15 respondents feel that the online materials are easy to navigate through and that resources can be found easily on the training hub, several users of the hub consider otherwise, hence improvements can be made in this direction. One other area which needs to be enhanced is the interaction feature between trainers/ organizers and trainees, as this around $33 \%$ of respondents either disagree or are not fully convinced that the hub provides enough opportunities to interact with the trainers/organizers so as to improve the learning experience. Overall the hub has been appreciated to perform well, to be user friendly, providing a good user experience.

For the Learning assessment, comparing pre and post-evaluation an increase in knowledge can be noticed, as the average score of 96.5 points recorded after the training with a standard deviation of \pm 4.5 points is 11.2 points greater than the one recorded before the training of 85.3 points with a standard deviation of \pm 11.8 points.

Table 1. Measures of tendency and variability pre and post-training

\begin{tabular}{|c|c|c|c|}
\hline & Score (pre) & Score (post) & $\begin{array}{c}\text { Absolute difference } \\
\text { (post-pre) }\end{array}$ \\
\hline Mean & 85.3 & 96.5 & 11.2 \\
\hline Percentile 25 & 78 & 94 & 16 \\
\hline Median & 88 & 100 & 4 \\
\hline Percentile 75 & 96 & 100 & 4 \\
\hline Mode & 96 & 100 & 28 \\
\hline Minimum & 60 & 88 & -28 \\
\hline Maximum & 100 & 100 & -12 \\
\hline Range & 40 & 12 & \\
\hline Inter-quartile range & 18 & 6 & \\
\hline Standard Deviation & 11.8 & 4.5 & \\
\hline
\end{tabular}

The distribution of scores is more spread out before the training, having a standard deviation of \pm 11.8 points, than after the training. Also, analyzing the interquartile range before and after the training it can be noticed that the distribution of scores for the pre-evaluation is more spread out than for the post-evaluation having a greater variability around the average score. Before the training $50 \%$ of the trainees scored between 96 points and 78 points, while after the training $50 \%$ of the trainees scored 94 points to 100 points.

Although an improvement in trainees' knowledge can be noticed based on the descriptive statistics, it has been tested whether there is a statistically significant before and after the training sessions. Wilcoxon Signed-Rank non-parametric test has been applied for testing if a statistically significant change occurred in the overall pre-evaluation scores and post-evaluation scores, hence if there has been an increase in knowledge.

H0: There is no change in the knowledge of trainees in the field of CSM after attending the ChemSMHUB training course.

H1: There has been a statistically significant change in the knowledge of trainees in the field of CSM after attending the ChemSM-HUB training course. 
Table 2. Wilcoxon Signed-Rank test - Hypothesis test summary

\begin{tabular}{|c|c|}
\hline column & V3 \\
\hline Test & Related-Samples Wilcoxon Signed Rank Test \\
\hline Sig. & 0.0154915019188735 \\
\hline Decision & Reject the null hypothesis. \\
\hline Null Hypothesis & The median of differences between Pre_test_points and \\
& Post_test_points equals 0. \\
\hline
\end{tabular}

Note: Asymptotic significances are displayed. The significance level is 0.05 .

Wilcoxon Signed-Rank showed that there was a significant difference $(\mathrm{z}=-6.864, \mathrm{p}=0.015)$ between the scores received before the training compared to the ones received after the training. This indicates that we have enough evidence to reject the null hypothesis and that the ChemSM-HUB pilot training contributed to the increase in knowledge of participants in the field of CSM. From the descriptive statistics - including the median values of 88 points before the training and 100 points after the training - and from the histograms of the distribution of scores it can be noticed that the change in knowledge has been a positive one and this translates into strengthen competencies in the field of CSM.

\section{Conclusions}

According to the feedback received in both cases - during peer review and the pilot training, materials and the hub have been subject to various changes and resulted in a comprehensive training package and, respectively, a training hub that facilitates individual study and certification of the DU \& D of chemicals, and provides access to up-to-date information in a concise and structured format. The complex aspects of the latest CSM legal requirements are thus simplified and described in terms understandable and useful to a broad audience of people responsible for chemical safety among DU and to D of chemicals who cannot afford the qualified CSM specialist in their company.

The Erasmus+ project reached its objective to deliver innovative vocational training on the elements of CSM, integrated with the e-learning hub and mobile application, dedicated mainly to employees responsible for CSM among downstream users (DU) and to distributor (D) of chemicals. Moreover, the training course has reached its objective to enhance "the competencies in the area of chemical safety management of downstream users (DU) and distributors (D) of chemicals across four countries.".

A comprehensive pack of instruments for information and training - printed and electronic format, has been developed and made available free of charge in five languages - English, Polish, German, Greek, and Romanian which contributed to reaching the objective to increase the awareness of relevant professional communities over the European legislation in the field, strengthen the target groups competences in the CSM field, and enhance the ability of trainers to create and deliver vocational training in the partnering countries.

The ChemSM-HUB website (address https://chemsm-hub.eu/hub/about.php) - is a virtual learning environment that not only enables users to pursue self-training and use the adequate assessment tools, but also brings free access to other available sources, enables the communication between learners and trainers, and serves as a central point for the exchange of information and ideas on CSM. The hub makes the information available to the largest possible audience, irrespective of the time, work, or place of residence. In other words, the ChemSM-HUB is a room for training and a room for assessment, as well as a facility for communication and networking.

The Mobile App for Android devices (address https://chemsm-hub.eu/dev/hub/trainingmobile.php) complements the training hub as it provides easy and quick access to find basic terms, definitions, and procedures related to the REACH and CLP Regulations available for anyone interested in general information and also for the ones who want a short and straight-ahead answer related to terms/ procedures.

The CSM training package has been designed on three successive levels - elementary notions, basic knowledge, and advanced knowledge - with available presentations and exercises organized in sessions on different topics, all explained step by step in the Training Patterns for Trainers and delivered in an interactive and friendly format on the e-learning platform embedded on ChemSM-HUB website. The 
training package and the training format proposed during the ChemSM-HUB project has contributed to a significant increase in the knowledge of chemical safety professionals.

And last but not least the Pocket Guide represents an important resource for downstream users and distributors of chemicals designed to help them meet the obligations imposed on companies under the REACH and CLP Regulations.

Acknowledgments: The authors would like to express their appreciation and thanks to all those who devoted their time and knowledge to the implementation of this project - special thanks to colleagues from Nofer Institute of Occupational Medicine, Łódź, Poland - Daria Pakulska, Dr., Sławomir Czerczak, Prof., and Ewa Michalak, M.Sc. Eng.; Lodz University of Technology, Łódź, Poland - Piotr Jóźwiak, Ph.D. Eng. and Grzegorz Szymański, Prof. Ph.D. Eng.; Oekopol GmbH, Germany - Olaf Wirth, Dr., and Isabela Banduch, Ph.D. Eng.; Prolepsis, Greece - Pania Karnaki, M.Sc., Matina Kouvari, M.Sc., and Ioannis Koutelidas, M.A..

\section{References}

1.2020 FACTS \& FIGURES of the European chemical industry, European Chemical Industry Council - Cefic aisbl, EU Transparency Register n 64879142323-90

2.MAROULIS, M., DE KETTENIS, P., BOUGAS, K., RAVET, J., REID, A., RZEPECKA, J., (VVA), Cumulative Cost Assessment for the EU Chemical Industry - Final Report, April - 2016, EUROPEAN COMMISSION, Directorate-General for Internal Market, Industry, Entrepreneurship and SMEs.

3.***A Roadmap to Revitalise REACH. REACH AUTHORISATION PROCESS A Critical Assessment, Dolores Romano and Tatiana Santos, European Environmental Bureau, November 2015 4.***The ChemSM-Hub project in the light of a new paradigm for chemical safety management, Daria Pakulska, Sławomir Czerczak, and Ewa Michalak, Nofer Institute of Occupational Medicine, Łódź, Poland, Department of Chemical Safety, International Journal of Occupational Medicine and Environmental Health 2020;33(3):247 - 261, https://doi.org/10.13075/ijomeh.1896.01562

5.REACH and CLP - the journey so far - SUMMARY, ECHA-16-A-03-EN

6.***Report on the Operation of REACH and CLP 2016, May 2016, European Chemicals Agency, ECHA-16-R-08-EN

7.***Work Programme 2017 - Highlights, ECHA-17-B-01-EN

8.KIRKPATRICK, D.L., KIRKPATRICK, J.D., Evaluating Programs: The Four Levels, 3rd edition, Berrett-Koehler Publishers, 2006

9.PHILLIPS J.J., STONE R.D., How to measure training results, 2000, McGraw-Hill Companies, ISBN 978-0-07-138792-7.

Manuscript received: 21.12 .2020 